\title{
INE, 30 años de estadísticas bibliotecarias
}

\author{
Andrés-Santos Barba Pérez, Ana Palacios Serrano, \\ Natalia Arroyo Vázquez y Francisco Javier Villoldo Gómez \\ Departamento de Análisis y Estudios \\ Fundación Germán Sánchez Ruipérez \\ Peñaranda de Bracamonte
}

\section{Resumen}

Hace 30 años que el INE viene publicando su serie de datos estadísticos sobre las bibliotecas en España. Los orígenes de esta publicación, el tipo de información recogida, los cambios de metodología adoptados y las variaciones en su presentación y contenidos se analizan en el presente artículo de cara a la mejor compresión de una de las herramientas fundamentales, por su antigüedad y amplitud, para el conocimiento y estudio de las bibliotecas Españolas en los tres últimos decenios.

\section{Las estadísticas bibliotecarias del INE}

A finales del año 2005 el Instituto Nacional de Estadística (INE) ha hecho público, a través de su sitio web (www.ine.es), los datos estadísticos de las bibliotecas en España del año precedente, concretamente del $2004^{1}$. Este acontecimiento se nos presenta en esta ocasión como un motivo para la celebración y la reflexión. Hace ya treinta años desde que el INE comenzara a publicar la serie denominada Estadística de Bibliotecas (en 1975 ve la luz el primer número con los datos estadísticos de 1973). Serie, sin embargo, no exenta de numerosos cambios, retoques y alguna que otra vicisitud, que marcaran su presencia y relevancia dentro de la estadística bibliotecaria.

Un repaso rápido a cómo y por qué surge, y a los periodos más significativos en la historia de estas estadísticas nos ayudará a conocer mejor el objeto y contenido de las estadísticas actuales, así como a valorarlas como una herramienta fundamental para el conocimiento histórico del conjunto de bibliotecas en España y el desarrollo de futuras líneas de política y

\footnotetext{
${ }^{1}$ http://www.ine.es/inebase/cgi/um?M=\%2Ft12\%2Fp403\%2Fa2004\&O=pcaxis\&N=\&L=0 (consultado el 01-06-
} 2006) 
planificación bibliotecaria, en aras a una gestión más racional y eficaz de los servicios bibliotecarios.

La publicación en 1975 de la Estadística de bibliotecas viene motivada por la recomendación de la UNESCO, en su decimosexta reunión celebrada en 1970 en París ${ }^{2}$, a los estados miembros para que en lo sucesivo adoptaran el modelo estadístico normalizado propuesto por su Conferencia General a fin mejorar la recogida y comparación internacional de los datos estadísticos bibliotecarios. El que esta publicación nazca a raíz de una 'obligación' adquirida con instituciones internacionales explica en parte el por qué de su escasa difusión y la ausencia de tradición analítica en el ámbito bibliotecario en España.

A la hora de establecer los periodos de análisis nos centraremos en el año de estudio estadístico y no en el año de su publicación. De acuerdo con este criterio podemos destacar tres periodos esenciales marcados por modificaciones significativas en el directorio de bibliotecas utilizado y en el cuestionario estadístico:

1. Primer periodo: de temporalidad anual, abarcaría, según se establece en la introducción de las estadísticas del año 1986, desde sus inicios, en 1973, hasta 1983. Entre 1978 y 1983 no se llegaron a publicar, únicamente apareció dentro del Anuario estadístico de España de estos años una tabla con los datos básicos bibliotecarios.

2. Segundo período: desde 1986 hasta 2000; pasa a ser bienal y se amplían sensiblemente los ítems recogidos. Entre 1983 y 1986, nos encontramos un vacío estadístico.

3. Tercer periodo: Desde 2002 hasta nuestros días. Se produce una importante modificación conceptual para adaptarse a la norma ISO 2789:1991: Estadística de las bibliotecas a nivel internacional. Mantiene su carácter bianual.

\section{Primer período: 1973-1983}

La adopción de las normas contenidas en la Recomendación sobre la normalización internacional de las estadísticas relativas a las bibliotecas, aprobada por la Conferencia General de la UNESCO en 1970, supone el comienzo de una nueva era en las estadísticas bibliotecarias.

\footnotetext{
${ }^{2}$ Recomendación sobre la normalización internacional de las estadísticas relativas a las bibliotecas. En UNESCO. General Conference; 16th; Actas de la Conferencia General, 16a reunión, París, 12 de octubre-14 de noviembre de 1970, v. 1: Resoluciones; 1971. Texto integro disponible en:

http://unesdoc.unesco.org/images/0011/001140/114046s.pdf\#page=144
} 
El esquema seguido en esta publicación se organiza en tres partes. Una primera de carácter conceptual denominada Introducción donde se fija el marco teórico de desarrollo (ámbito de estudio, definiciones y clasificación de bibliotecas) y la metodología seguida en la recogida de datos (explicación de los ítems incluidos); una segunda parte donde se recogen los datos obtenidos, agrupados en el resumen nacional y en las tablas provinciales; y por último, un anexo donde se ofrece el cuestionario empleado.

En la Introducción se siguen, casi al pie de la letra, las indicaciones de la citada Recomendación. Señalar, como más significativos, los siguientes aspectos:

- Ámbito de estudio: definido específicamente para las bibliotecas españolas. "Esta investigación estadística se extiende a todas las bibliotecas radicadas en el territorio nacional, cualquiera que sea su dependencia administrativa, excepto las bibliotecas privadas — de particulares o entidades— cuya utilización no está permitida al público ni siquiera en condiciones especiales".

- Definiciones: resulta curiosa la definición de biblioteca recogida. "Biblioteca: sea cual fuere su denominación, es toda colección organizada de libros y publicaciones periódicas impresas o de cualesquiera otros documentos, en especial gráficos o audiovisuales, así como los servicios del personal que facilite a los usuarios la utilización de estos documentos con fines informativos, de investigación, de educación o recreativos." Definición que a pesar de estar centrada en la colección, enfoque actualmente en desuso, resulta ciertamente avanzada para la época por la inclusión que hace de los materiales no librarios. Sin embargo, la Recomendación, y por extensión el cuestionario del INE, no contemplan la recogida de estos datos, centrándose únicamente en estos tres grupos: libros y publicaciones periódicas, manuscritos y microcopias.

- Clasificación de las bibliotecas: se indica que se sigue la clasificación determinada por la UNESCO adaptándola a la organización bibliotecaria española. En esencia no hay prácticamente ninguna diferencia, exceptuando que se omite el grupo de "Otras bibliotecas importantes no especializadas: bibliotecas no especializadas, de carácter científico o erudito, que ni son universitarias, ni nacionales, aunque puedan ejercer funciones de biblioteca nacional en un área geográfica determinada" debido a que en la concepción de estado centralizado de la época éstas no tenían cabida. Por tanto, los tipos de bibliotecas recogidos son: bibliotecas nacionales, bibliotecas de instituciones de enseñanza superior, bibliotecas escolares (que en los sucesivos periodos desaparecerán), bibliotecas especializadas y bibliotecas públicas o populares. Elocuente es la definición que se da de bibliotecas públicas y, fundamentalmente, en cuanto a las categorías de usuarios que cita, que sorprendentemente se mantendrá 
hasta el año 2002: "Bibliotecas públicas (o populares): bibliotecas que están, gratuitamente o por módica suma al servicio de una comunidad local o regional, para atender al público en general, o a ciertas categorías de usuarios como niños, militares, enfermos de los hospitales, presos, obreros y empleados". En este primer periodo se omite la diferenciación propuesta en la Recomendación entre: "las bibliotecas públicas propiamente dichas, es decir, las bibliotecas financiadas totalmente o en su mayor parte, por los poderes públicos (bibliotecas municipales o regionales) y las bibliotecas financiadas con fondos privados".

El modelo de cuestionario se hace atendiendo igualmente a las indicaciones de la Recomendación y tiene como finalidad el estudio de los distintos tipos de bibliotecas y de sus datos fundamentales de uso y funcionamiento. El número de ítems es bastante reducido, nueve, a los que hay que añadir los datos identificativos de la biblioteca y el número de puntos de servicio. Estos ítems son: Población servida, Fondos de la biblioteca, Prestatarios inscritos, Personal de la biblioteca (estableciendo, en base a la Recomendación, una diferenciación entre diplomados y no diplomados en Biblioteconomía y el parámetro de personal en equivalencia en jornada completa), Número de adquisiciones, Título de publicaciones periódicas en curso recibidas durante el año, Préstamos (además de a domicilio, a otras bibliotecas del país o internacionales), Fotocopias y otras copias y Gastos (gastos ordinarios y de capital, lo que identificamos actualmente como corrientes y de inversión).

\section{Segundo periodo 1986-1998}

Con la publicación de un folleto con los datos anticipados ${ }^{3}$ de 1986 el INE inicia una modificación de las estadísticas motivada, según se explica en la introducción de la publicación de los datos estadísticos de 1988, "tanto por la necesidad de subsanar el deterioro que se produce a través de los años en una estadística continua, como por la urgencia de acometer su automatización".

También se cita en esta publicación como principales modificaciones acometidas, las siguientes:

1. Ampliación de las características que se solicitan a todas las bibliotecas del país (excepto las escolares) fundamentalmente en lo referente a su situación, equipamiento y funcionamiento.

\footnotetext{
3 “Estadística de Bibliotecas : año 1986. Datos anticipados”. Madrid: Instituto Nacional de Estadística, 1988. 12 p.
} 
2. Actualización del directorio ${ }^{4}$

3. Modificación del cuestionario atendiendo a las recomendaciones de la UNESCO

4. Estadística bianual

5. Realización de la estadística de las bibliotecas escolares en una fase posterior recogiéndose por muestreo las características de estas bibliotecas a través de un cuestionario simplificado, y utilizándose como marco la información solicitada a través de la Estadística de la Enseñanza ${ }^{5}$.

6. Tratamiento informático de los datos

El esquema de la publicación de esta serie (excluyendo el folleto con el avance de resultados de 1986), aunque en esencia mantiene las características del periodo anterior, varia ligeramente, diferenciando únicamente dos partes el corpus teórico o metodológico, dividido en Introducción, Objetivos, Ámbito de la estadística... y Cuestionario utilizado, y la parte estadística con las tablas de resultados agrupadas en tablas nacionales y tablas por comunidades autónomas y provincias. Se añade al final una tabla para recoger las incidencias de las bibliotecas que no respondieron al cuestionario.

En lo que hemos denominado 'corpus teórico' y bajo el epígrafe de Características de las bibliotecas se hace una distinción entre las características consideradas estructurales (datos para la identificación de la biblioteca, funcionamiento y dotación) y aquellas que reflejan la actividad anual derivada del trabajo bibliotecario (fondos, préstamos, prestatarios...). Clasificación que sigue utilizándose.

El cuestionario sufre una importante remodelación. Los veintiún ítems recogidos se agrupan en: Clase de biblioteca, Funcionamiento, Equipamiento, Fondos y movimiento, Actividad, Personal y Gastos.

Resaltar las siguientes modificaciones:

- A fin de facilitar la recogida de datos, se enumeran las categorías contempladas en el tipo de biblioteca (Nacional, Especializada universitaria, Especializada de instituciones religiosas, Otras especializadas, Pública de carácter general, Pública de hospitales, Pública de organismos militares, Pública de colegios mayores, Pública de instituciones

\footnotetext{
${ }^{4}$ Se procede a actualizar el directorio de bibliotecas controlado por las delegaciones provinciales del INE con las bibliotecas procedentes del Censo-Guía de Bibliotecas de la Dirección General del Libro y Bibliotecas del Ministerio de Cultura, así como con el directorio de Bibliotecas Especializadas, Universitarias y de Organismos Públicos del Servicio de Fondos Bibliográficos del Ministerio de Educación y Ciencia y con la actualización de las bibliotecas públicas que pasan a depender de las comunidades autónomas.

${ }^{5}$ En teoría las bibliotecas escolares serían objeto de un proyecto y explotación específicos, algo que nunca llegaría a realizarse
} 
penitenciarias, y Otras públicas, a especificar)6 y los órganos de los que dependen (Administración Central, Autonómica, Local, Instituciones privadas sin ánimo de lucro u Otro).

- Se añade el año de inicio del funcionamiento de la biblioteca.

- En cuanto al personal, se hace una nueva distinción entre Bibliotecarios profesionales, Bibliotecarios formados en el ejercicio de sus funciones, (diferenciación motivada por la práctica ausencia de titulados en Biblioteconomía y la abundancia de personal que con otras titulaciones venía desempeñando este cargo) y Otro personal.

- Se incluyen nuevos datos. Se pregunta por primera vez por el número de actividades culturales organizadas y desarrolladas por las bibliotecas, por el tipo y número de aparatos reproductores (fotocopiadoras, lectores de microfichas, microfilms, impresoras u otros), el número de reprografías y si la biblioteca está automatizada o en proyecto. También se empieza a recoger información sobre los documentos audiovisuales.

A lo largo de este periodo el cuestionario va sufriendo pequeñas modificaciones, que recogeremos de forma resumida. Así en el año 1990, se reorganiza el apartado de tipos de bibliotecas (agrupándolas bajo los epígrafes Nacional, Otras importantes no especializadas - recogida desde el inicio en la Recomendación de la UNESCO pero no contemplada hasta este momento-, Públicas o populares, Instituciones de enseñanza superior y Especializadas, estas tres últimas subdivididas en sus respectivas categorías) y se añade en el apartado de Titularidad la de Universitaria. En el cuestionario se integra el número de documentos dados de baja, por expurgo, pérdida, etc., y se incluye la posibilidad de indicar el material informático dentro del ítem Otros en los aparatos de reproducción (tanto el material como el equipo informático tendrán en el cuestionario de 1994 una entrada propia y diferenciada).

Una novedad muy interesante en la publicación de los datos de 1990, que desaparece en años posteriores, es la inclusión de un apartado titulado Análisis de resultados que estudia la información recogida, en que el texto se acompaña con tablas y gráficos explicativos.

En 1996 se confeccionan cuestionarios bilingües para la recogida de datos de las bibliotecas situadas en comunidades autónomas con lenguas descritas como vernáculas. En 2004 pasan a denominarse cooficiales.

\footnotetext{
${ }^{6}$ Como puede apretarse, todavía se mantiene dentro de las bibliotecas públicas un grupo amplio de bibliotecas cuyo carácter no es propiamente público, de libre acceso a cualquier ciudadano.
} 
En el 2000, a propuesta del Grupo de Trabajo de Estadísticas de Bibliotecas Públicas ${ }^{7}$ se presta una especial atención a la integración del mundo informático y de las telecomunicaciones en las bibliotecas incorporando en el cuestionario algunas cuestiones referidas a los servicios y funciones asociados a Internet. Dentro de los aparatos de reproducción se establece la diferenciación entre los terminales informáticos de uso interno y de uso público, para los usuarios. Se incluye un epígrafe nuevo "Internet y servicios al y del exterior" en el que se consignará la conexión a Internet, la disponibilidad en la biblioteca de correo electrónico y página web, la disponibilidad del catálogo (manual, CD-ROM, red local, Internet...) y en el servicio de reprografía se incluye el número de impresiones de publicaciones electrónicas. Aparece un nuevo ítem, el Número estimado de usuarios, que se corresponderá con los visitantes en sucesivos cuestionarios. En cuanto a la publicación de las estadísticas, decir que desaparece la publicación impresa quedando únicamente disponible en formato electrónico (disquete, CD-ROM y web). Este año supone el inicio de la publicación en web de las Estadísticas de bibliotecas.

\section{Tercer periodo: a partir de 2002}

Marcado por la actualización del directorio de bibliotecas, que va a suponer un descenso en el número de bibliotecas, de 7.103 unidades administrativas (8.208 puntos de servicio) en 2000 se pasa a 6.371 (7.939) en 2002, y la adopción de la norma ISO 2789:1991, fruto de la colaboración con el Grupo de Trabajo de Estadísticas de Bibliotecas Públicas.

Los cambios y novedades más importantes son:

- La adaptación al concepto de biblioteca recogido en la normal ISO 2789: "Se entenderá por biblioteca, toda organización o parte de una organización, cuyo fin principal es reunir y mantener una colección organizada de documentos y facilitar el uso de los recursos de información a fin de satisfacer las necesidades informativas, de investigación, educativas, culturales o recreativas de los usuarios".

- Las modificaciones en la tipología bibliotecaria (se añaden las Bibliotecas Centrales de Comunidades Autónomas y las Bibliotecas para grupos específicos de usuarios —no especializadas-) y los requisitos que ha de cumplir una biblioteca pública ${ }^{8}$.

\footnotetext{
${ }^{7}$ Grupo de Trabajo que se crea en 1997 constituido por participantes del Ministerio de Cultura y de las Consejerías de Cultura de las Comunidades Autónomas y cuya finalidad es la de llegar a obtener unas estadísticas fiables de las bibliotecas públicas en España. Para ello trabaja en el desarrollo de un formulario normalizado y común para todas las comunidades adecuado a los estándares internacionales. En relación con el INE se establece una línea de colaboración de cara a coordinar sus respectivas iniciativas estadísticas.

8 "Una biblioteca es pública cuando cumple los siguientes criterios:

- Dispone de una colección de fondos de carácter general, ofrecen servicios informativos de tipo cultural, educativo, recreativo y social y son accesibles a todos los ciudadanos.

- Ofrece los servicios de préstamo y consulta de documentos de forma libre y gratuita.

- Los fondos de las bibliotecas públicas son de libre acceso y susceptibles de ser dejados en préstamo.
} 
- Se suprime la información sobre el año de inicio de funcionamiento de la biblioteca y se incluye la forma de acceso a las bibliotecas, libre o restringida.

- Se varía la tipología de los fondos y la del personal y se introduce el recuento de visitantes ${ }^{9}$.

- Se contabilizan los volúmenes anteriores a 1901, y los servicios de consulta en sala.

- En el apartado de Equipamiento físico e informático, además de solicitar el número de ordenadores y terminales, se solicita el número de impresoras y escáneres. Se hace mayor hincapié en los servicios de Internet, se pregunta por el número de ordenares y terminales conectados, por si este servicio es de pago, y, en caso de que la biblioteca tenga página web, por el número de visitantes.

- Se cambia el término de Prestatarios inscritos por el de Usuarios inscritos, más adecuado, y se diferencia entre adultos e infantiles.

- Dentro del servicio de consulta en sala se pregunta por la posibilidad de consulta de documentos audiovisuales, sonoros y electrónicos.

- En cuanto a los préstamos, se empiezan a pedir diferenciados para cada el tipo de documento.

- El apartado de Personal se vuelve a reestructurar en dos grupos, personal incluido en plantilla y personal no incluido, según una clasificación fijada por categorías.

- Finalmente, en Gastos se incorporan los Gastos de inversión.

Estadística de bibliotecas: 1973-2004. Resumen de resultados

\begin{tabular}{|l|r|r|r|r|r|}
\hline & \multicolumn{1}{|c|}{1973} & \multicolumn{1}{c|}{1988} & \multicolumn{1}{c|}{2000} & \multicolumn{1}{c|}{2002} & \multicolumn{1}{c|}{2004} \\
\hline Puntos de Servicio & 3.700 & 6.169 & 8.208 & 8.099 \\
\hline $\begin{array}{l}\text { Prestatarios } \\
\text { Inscritos }\end{array}$ & 1.307 .918 & 9.527 .777 & 13.605 .730 & 12.595 .917 & 13.884 .650 \\
\hline Fondos* & 23.861 .956 & 70.397 .488 & 141.991 .982 & 179.018 .657 & 201.458 .068 \\
\hline Libros & 23.506 .597 & 57.188 .319 & 110.256 .128 & 117.609 .196 & 132.144 .564 \\
\hline $\begin{array}{l}\text { Préstamos a } \\
\text { usuarios }\end{array}$ & 5.323 .882 & 23.706 .543 & 47.751 .587 & 55.753 .166 & 65.727 .309 \\
\hline Personal** & 6.012 & 13.351 & 22.855 & 21.072 & 22.665 \\
\hline Personal ETC*** & $3.995,00$ & $9.716,04$ & $17.786,26$ & $17.139,54$ & $18.387,57$ \\
\hline Gastos corrientes & $3.496 .081 €$ & $202.744 .107 €$ & $640.194 .980 €$ & $599.997 .923 €$ & $694.628 .447 €$ \\
\hline
\end{tabular}

* Número total de fondos existentes en las bibliotecas de acuerdo a las categorías que se recogen en cada año estadístico

** Personal: en los años 2002 y 20004 sólo se contabiliza el personal incluido en platilla

*** Personal en equivalente a tiempo completo. En los años 2002 y 20004 sólo se contabiliza el personal incluido en platilla

Fuente: "Estadística de Bibliotecas". 1973, 1988, 2000, 2002 y 2004

No obstante, cuando es necesario por razones de seguridad y conservación, pueden limitar el acceso a una parte de estos fondos."

${ }^{9}$ En realidad lo que se recoge bajo la especificación de Visitantes se aproxima más al número de visitas que recibe la biblioteca que al número de personas que la visitan. 
De la publicación de los datos estadísticos del año 2004 únicamente están disponibles en el sitio web del INE los resultados detallados, por lo que no podemos completar el análisis de la publicación en cuanto a su metodología, pero comparando los cuestionarios de 2002 y 2004 podemos decir que no existe ninguna novedad, ambos son idénticos.

\section{Bibliografía}

Estadística de bibliotecas. Madrid: Instituto Nacional de Estadística. 1973-2004. Serie irregular (de 1973 a 1977 de carácter anual y a partir de 1986, bianual). Desde el año 2000 está disponible en http://www.ine.es/inebase/cgi/um?M=\%2Ft12\%2Fp403\&O=inebase

Hernández Sánchez, Hilario. "Las bibliotecas públicas en España. Una realidad abierta". En: Las bibliotecas públicas en España. Una realidad abierta. Madrid: Fundación Germán Sánchez Ruipérez, 2001. Pp. 17-133. Consultado en 01-06-2006.

http://www.fundaciongsr.es/bp/bp01_1-1.htm

"Recomendación sobre la normalización internacional de las estadísticas relativas a las bibliotecas". En: UNESCO. General Conference; 16th; Actas de la Conferencia General, 16a reunión, París, 12 de octubre-14 de noviembre de 1970, v. 1: Resoluciones; 1971. Consultado en: 01-06-2006. http://unesdoc.unesco.org/images/0011/001140/114046s.pdf\#page=144

Selgas Gutiérrez, Joaquín: "Las estadísticas de bibliotecas públicas en España: pasado, presente y perspectivas de futuro". En: La Biblioteca Pública: Compromiso de Futuro. Actas del II Congreso Nacional de Bibliotecas Públicas. Salamanca, 17, 18 y 19 de noviembre de 2004. Madrid: Ministerio de Cultura, 2004. Pp. 172-181. Consultado en: 01-06-2006. http://travesia.mcu.es/documentos/congreso_2bp/1a_sesion/comunicacion17.pdf 